\title{
Kidney volume and function in autosomal dominant polycystic kidney disease
}

\author{
Eiji Higashihara $\cdot$ Kikuo Nutahara $\cdot$ Takatsugu Okegawa $\cdot$ \\ Toshihide Shishido $\cdot$ Mitsuhiro Tanbo $\cdot$ Kuninori Kobayasi • \\ Toshiaki Nitadori
}

Received: 15 April 2013/Accepted: 20 June 2013/Published online: 18 July 2013

(C) The Author(s) 2013. This article is published with open access at Springerlink.com

\begin{abstract}
Background The significance of total kidney volume (TKV) as a biomarker of kidney function in autosomal dominant polycystic kidney disease (ADPKD) is controversial and has been reappraised.

Methods Between 2007 and 2012, 64 patients were followed with a mean 39.7-month observation period. TKV measurements by magnetic resonance imaging and estimation of renal function with estimated glomerular filtration rate (eGFR) using the Modification of Diet in Renal Disease equation and 24-h urine creatinine clearance were repeated annually.

Results TKV and its adjusted parameters (height-adjusted, body surface area-adjusted and log-converted TKV $[\log -\mathrm{TKV}])$ correlated with eGFR significantly. Among them, the correlation coefficient of log-TKV was most significant $(r=-0.6688, p<0.001)$. The eGFR slope correlated negatively with TKV slope $(p<0.05)$. TKV increased faster and became larger as chronic kidney disease (CKD) stage advanced. As age advanced, eGFR declined significantly $(p<0.001)$, but the eGFR slope remained constant. There was no significant correlation between TKV and age, but the log-TKV slope became
\end{abstract}

\footnotetext{
E. Higashihara $(\square)$

Department of ADPKD Research, Kyorin University School of Medicine, 6-20-2 Shinkawa, Mitaka, Tokyo 181-8611, Japan

e-mail: ehigashi@ks.kyorin-u.ac.jp

E. Higashihara $\cdot$ K. Nutahara $\cdot$ T. Okegawa $\cdot$ T. Shishido ·

M. Tanbo

Department of Urology, Kyorin University School of Medicine, Mitaka, Tokyo, Japan

K. Kobayasi · T. Nitadori

Department of Radiology, Kyorin University School of Medicine, Mitaka, Tokyo, Japan
}

smaller as age advanced. If baseline TKV was large, the eGFR slope was steeper $(p<0.05)$, which suggests that eGFR declines faster in patients with larger kidney volume. Conclusions TKV is confirmed as a clinically meaningful surrogate marker in ADPKD. Log-TKV correlates with eGFR most significantly. Higher rates of kidney enlargement and larger kidney volume are associated with a more rapid decrease in kidney function. Kidney function decreased faster as CKD stage advanced, but its declining slope did not change significantly by age, at least after $\sim 30$ years of age.

Keywords Autosomal dominant polycystic kidney disease $\cdot$ Glomerular filtration rate $\cdot$ Kidney volume

\section{Introduction}

Autosomal dominant polycystic kidney disease (ADPKD) is the most common hereditary kidney disease characterized by the progressive enlargement of innumerable renal cysts that lead to the deterioration of kidney function [1-3]. The Consortium for Radiologic Imaging Studies of Polycystic Kidney Disease (CRISP) study showed that baseline total kidney volume (TKV) predicted the subsequent rate of an increase in volume, independently of age [4]. Higher rates of kidney enlargement are associated with a more rapid decrease in renal function.

In a more recent study on CRISP participants, heightadjusted TKV (ht-TKV) predicted the risk of developing renal insufficiency in ADPKD patients within 8 years of follow-up [5]. The reason for adopting ht-TKV as an adjusted TKV marker in this study was to minimize the differences in adjusted TKV values between men and women. Other adjusted TKV markers, such as body 
surface-adjusted TKV (bs-TKV) or log-converted TKV $(\log -\mathrm{TKV})$, were compared from the standpoint of minimizing the differences between men and women. It remains unclear which adjusted TKV marker correlates best with renal function.

On the other hand, the results from three recent prospective clinical trials examining the effect of mammalian target of rapamycin inhibitors on disease progression of ADPKD have not demonstrated an association between changes in TKV and glomerular filtration rate (GFR) [6-8]. These studies might have used too short a period for examining the relationship between TKV and functional changes.

If TKV correlates with kidney function, it will be a useful clinical marker of renal function since (1) it can be measured reliably, and (2) it changes by a measurable amount during a relatively short period of time (mean \% increase of TKV is 5-6\% per year) [9]. In contrast, kidney function, measured by estimated GFR (eGFR), decreases at a slow rate of $0-3 \mathrm{ml} / \mathrm{min} / 1.73 \mathrm{~m}^{2}$ per year depending on the chronic kidney disease (CKD) stage [10]. Taking the measurement variation of eGFR into consideration, it is difficult to detect a small change as significant, especially during early CKD stages when a relatively small amount of eGFR decreases from a relatively large baseline eGFR.

For the above reasons, we reappraised the relationship between kidney volume and kidney function (using eGFR). If a significant relationship between two parameters was confirmed, the characteristics of these parameters and their changes (slopes) in relation to age and CKD stage were examined.

\section{Materials and methods}

All patients fulfilled Ravine's diagnostic criteria of ADPKD. One hundred and eighty-eight patients with ADPKD gave informed consent to take part in an observational clinical study protocol measuring TKV once a year with simultaneous collection of 24-h urine for determination of creatinine clearance (Ccr) and urinary protein excretion between April 2007 and July 2012. Patients with end-stage renal disease (ESRD) underwent TKV measurement only. Of 188 patients, 70 underwent TKV measurement three times or more. Two patients who received laparoscopic cyst fenestration, one patient with a ureteral stone with hydronephrosis during the study period, and three patients with baseline ESRD were excluded from analysis.

Serum creatinine was measured enzymatically. Kidney function was estimated with Ccr using 24-h urine, reciprocal creatinine and eGFR. eGFR was calculated using the following formula-eGFR (male) $=194 \times \mathrm{Cr}^{-1.094} \times$ $\mathrm{Age}^{-0.287}$, and eGFR (female) $=\mathrm{eGFR}($ male $) \times 0.739$.
This equation is a Japanese coefficient of the modified Isotope Dilution Mass Spectrometry-Modification of Diet in Renal Disease (IDMS-MDRD) Study [11]. The staging of kidney function is based on the Kidney Disease Outcomes Quality Initiative Clinical Practice Guidelines for CKD [12] using the final eGFR measurement.

TKV was measured by high-resolution magnetic resonance imaging (MRI) using a volumetric measurement of cross-sectional imaging, as described in the report from the CRISP study [13]. Gadolinium enhancement was not used for safety reasons. TKV was adjusted by height (ht-TKV, $\mathrm{ml} / \mathrm{m}$ ), body surface area (bs-TKV, $\mathrm{ml} / \mathrm{m}^{2}$ ) and log-converted form $(\log -\mathrm{TKV}, \log [\mathrm{ml}])$. Kidney volume was measured by one radiologist (KK). Intrareader reliability was extremely high-the correlation coefficient was 0.999 for ten different single kidney volume measurements at different times when blind to first measurement. The mean of the \% difference between two measurements was $0.29 \pm 3.28(\mathrm{SD}) \%$.

Twenty-four-hour urinary protein excretion was expressed as the mean value of several measurements for each patient. The slopes of TKV, adjusted TKV parameters and kidney function parameters were calculated using linear regression analysis for each patient. \%TKV was calculated with baseline TKV as $100 \%$.

The study protocol was approved by an institutional review board (09-56), and the study was conducted in accordance with the guidelines of the Declaration of Helsinki. All participants gave written informed consent to use their clinical data for medical research.

\section{Statistical analyses}

Analyses were performed with StatMate 4 and SAS 10 for Windows. Parametric variables are expressed as the mean and standard deviation in parentheses. Two-sided $p<0.05$ was considered to indicate statistical significance. $p$ values for differences between CKD stages were obtained using ANOVA or the Kruskal-Wallis test. Correlations between two variables were examined by linear regression analysis. The correlation coefficient $(r)$ was obtained by the Spearman rank-order correlation coefficient.

\section{Results}

Between April 2007 and July 2012, 188 patients with ADPKD attending our clinic were followed annually by measuring TKV with MRI and 24-h urine collection. Among them, 70 patients repeated MRI and 24-h urine measurements three times or more. Six patients with a medical history affecting kidney volume, such as laparoscopic fenestration and baseline ESRD, were excluded 
from the study, leaving 64 patients for analysis (67\% were female).

Four of the 64 patients had ESRD and one died of cerebral hemorrhage during this observation period. Baseline characteristics and the annual change rate (slope) of kidney function and volume are shown in Table 1. Mean slope of $\% \mathrm{TKV}$ and eGFR were $5.9 \%$ per year and $-1.0 \mathrm{ml} / \mathrm{min} / 1.73 \mathrm{~m}^{2}$ per year, respectively.

Relationship between TKV and kidney function

TKV, ht-TKV, bs-TKV and log-TKV are all significantly correlated with eGFR (Fig. 1). Figure 1 illustrates the data measured at final observation, but qualitatively similar results were obtained using baseline observation. Among these parameters, log-TKV correlation was most significant. Baseline TKV and ht-TKV, but not bs-TKV and logTKV, negatively correlated with the eGFR slope $(r=$ $-0.2642,-0.2476,-0.1811$ and $-0.2425, p=0.0349$, $0.0485,0.1521,0.0534$, respectively, Fig. 2a). There was a weak but significant correlation between the eGFR slope and TKV slope ( $r=-0.2593, p=0.03853$, Fig. $2 b)$.

Table 1 Baseline and annual change rate (slope) data of kidney volume and function

\begin{tabular}{|c|c|}
\hline$N$ (men/women) & $64(21 / 43)$ \\
\hline Age (year) & $47.0(14.1)$ \\
\hline Observation period (months) & $39.7(11.1)$ \\
\hline \multicolumn{2}{|c|}{ Baseline data of kidney volume and function } \\
\hline TKV (ml) & $1,681.1(1,001.1)$ \\
\hline ht-TKV (ml/m) & $1,023.8(604.2)$ \\
\hline bs-TKV $\left(\mathrm{ml} / \mathrm{m}^{2}\right)$ & $1,029.4(615.2)$ \\
\hline $\log -\mathrm{TKV}(\log [\mathrm{ml}])$ & $3.1588(0.2357)$ \\
\hline $1 / \mathrm{Cre}(\mathrm{ml} / \mathrm{mg})$ & $109.8(42.7)$ \\
\hline eGFR $\left(\mathrm{ml} / \mathrm{min} / 1.73 \mathrm{~m}^{2}\right)$ & $60.2(27.38)$ \\
\hline $\operatorname{Ccr}\left(\mathrm{ml} / \mathrm{min} / 1.73 \mathrm{~m}^{2}\right)$ & $90.01(36.96)$ \\
\hline \multicolumn{2}{|c|}{ Annual change rate $\left(\right.$ slope,$\left.b^{*}\right)$ of kidney volume and function } \\
\hline TKV slope (ml/year) & $109.5(123.8)$ \\
\hline$\% \mathrm{TKV}$ slope (\%/year) & $5.90(4.38)$ \\
\hline ht-TKV slope (ml/m/year) & $65.9(74.4)$ \\
\hline bs-TKV slope (ml/m²/year) & $64.3(71.6)$ \\
\hline $\log$-TKV slope $(\log [\mathrm{ml}] /$ year$)$ & $0.022(0.021)$ \\
\hline 1/Cre slope (ml/mg/year) & $-0.948(8.073)$ \\
\hline eGFR slope $\left(\mathrm{ml} / \mathrm{min} / 1.73 \mathrm{~m}^{2} /\right.$ year$)$ & $-1.020(3.632)$ \\
\hline Ccr slope $\left(\mathrm{ml} / \mathrm{min} / 1.73 \mathrm{~m}^{2} /\right.$ year$)$ & $-3.753(9.233)$ \\
\hline
\end{tabular}

Numbers are the mean and standard deviation (in parentheses).

*A linear regression line $(y=a+b X)$ was obtained by regression analysis between each parameter and age (months) for the measurement of each patient and $b$ is expressed as change rate per year (slope)

$T K V$ total kidney volume, $h t-T K V$ TKV divided by height $(\mathrm{m}), b s$ $T K V$ TKV divided by body surface area $\left(\mathrm{m}^{2}\right), \log -T K V \log$-converted TKV, $e G F R$ estimated glomerular filtration rate by Japanese MDRD equation, $C \mathrm{cr}$ creatinine clearance measured by 24 -h urine collection
Statistically significant correlations between eGFR and TKV-related parameters support the view of a clinically meaningful surrogate marker of TKV in ADPKD. The significant correlation between baseline TKV and eGFR slope (Fig. 2a) suggests the prognostic value of TKV for kidney functional deterioration.

TKV and function in relation to CKD stage

Individual data plotted as age-related TKV according to different CKD stages (Fig. 3) and Table 2 show that TKV increases faster and becomes larger as CKD stages advance. Age, systolic blood pressure, proteinuria, TKV, and TKV slope increase while eGFR slope decreases significantly $(p<0.001)$ as CKD stage advances (Table 2). Stages 1 and 2 are combined because TKV did not differ significantly $(1264 \pm 511 \mathrm{ml}$ in stage $1(n=7)$ and $1492 \pm 595 \mathrm{ml}$ in stage $2(n=24), p=0.3666)$.

In five of seven patients with CKD stage 5, TKV increased $>3,000 \mathrm{ml}$. In contrast, only two of 46 patients with CKD stages 1-3 had TKV $>3,000 \mathrm{ml}$ (Fig. 1, $p<0.001)$.

In patients with advanced CKD stages, eGFR decreased faster, which was demonstrated by a significant correlation between final eGFR and the eGFR slope $(r=0.4002$, $p=0.0011)$; however, no significant correlation was observed between baseline eGFR and the eGFR slope ( $r=0.1069, p=0.4007)$. There was a high correlation between baseline as well as final TKV and the TKV slope $(r=0.7995$ and $0.8955, p<0.001 p<0.001$, respectively), suggesting that patients with large kidneys have a rapid rate of kidney enlargement.

Changes in kidney volume and function in relation to age

As age advanced, eGFR, reciprocal creatinine and $\mathrm{Ccr}$ decreased significantly (Table 3 ). There was highly significant correlation between age and eGFR but the eGFR slope did not change significantly in relation to age.

TKV and TKV-related parameters had no significant correlation with age (Fig. 5). In contrast, the \% TKV slope and log-TKV slope became smaller as age advanced (right panel of Table 3 and Fig. 5d). There was no significant correlation between function-related slopes and age.

The age-related results were not qualitatively different between baseline and final age.

\section{Discussion}

The present study confirmed the significant relationship between TKV and kidney function, which was reported by 
(A) eGFR and total kidney volume

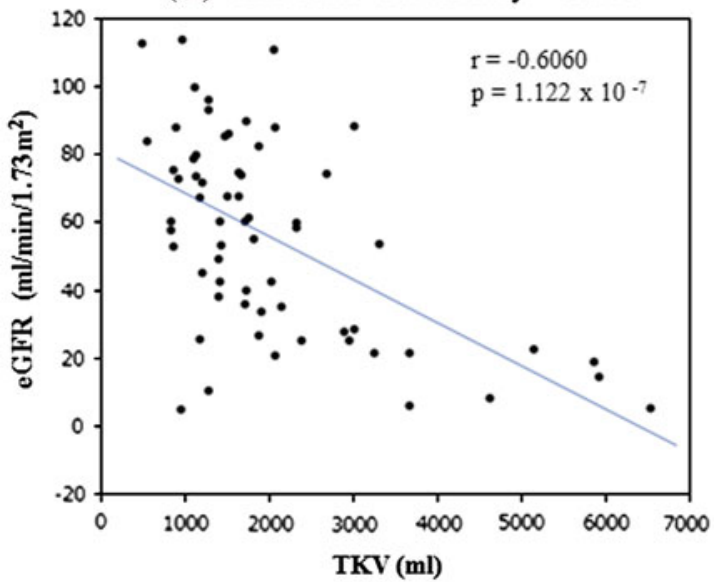

(C) eGFR and body surface area-adjusted TKV

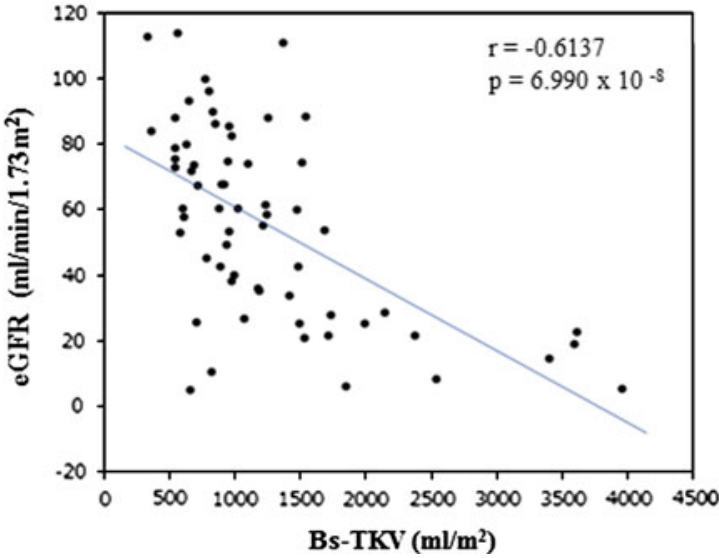

Fig. 1 Correlation of a total kidney volume (TKV), b height-adjusted TKV (ht-TKV), c body surface area-adjusted TKV (bs-TKV) and d log-converted TKV (log-TKV) to estimated glomerular filtration
(B) eGFR and height adjusted-TKV

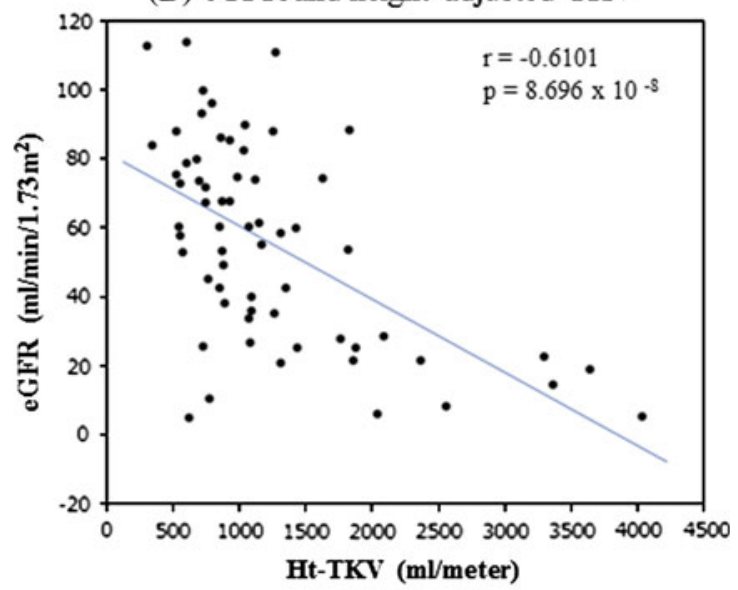

(D) eGFR and log-converted TKV

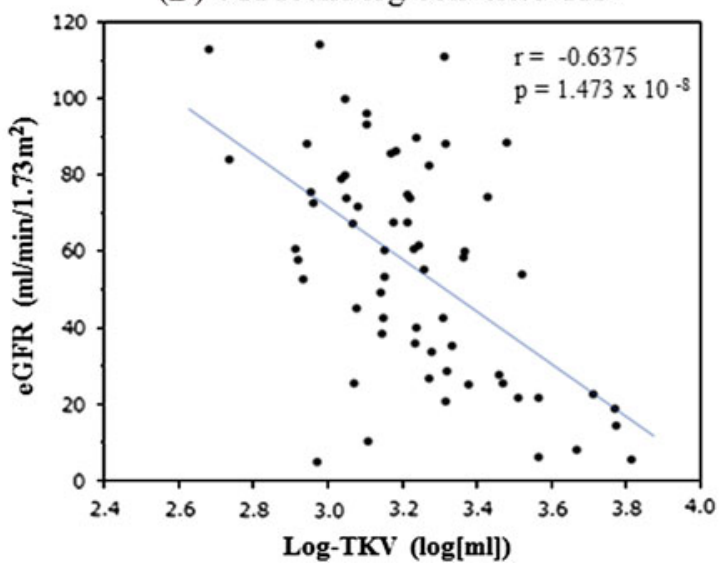

rate (eGFR). These values are final measurements. The correlation coefficients $(r)$ of all TKV-related parameters are significant. Among them, $r$ of log-TKV is most significant
(A) Baseline TKV and eGFR slope

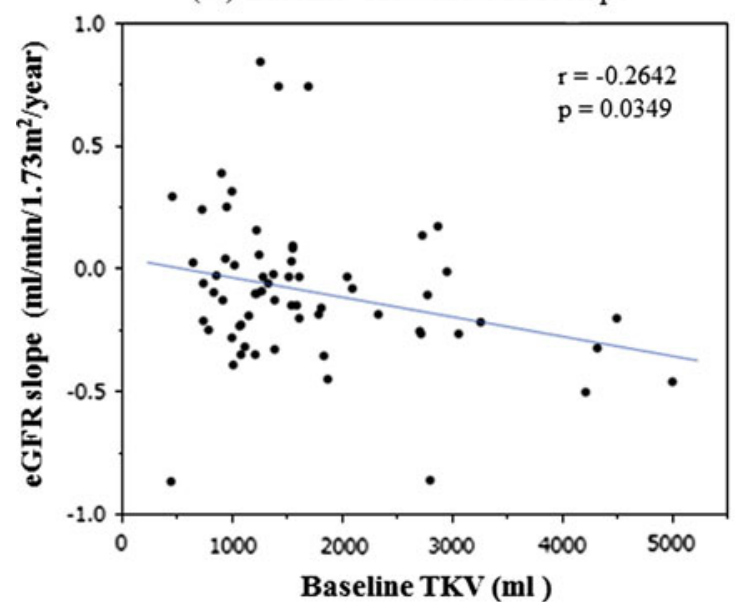

(B) Slopes of TKV and eGFR

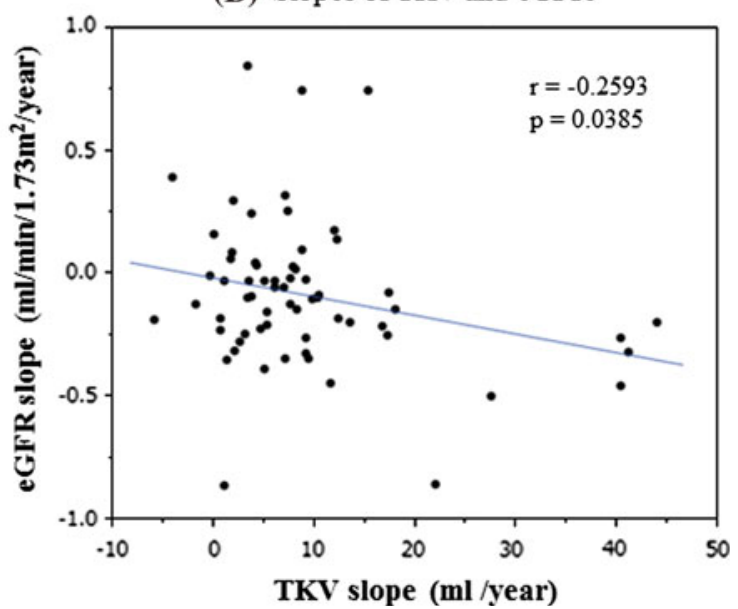

Fig. 2 a Correlation coefficient $(r)$ between baseline TKV and eGFR slope is significant $(p=0.0349)$. b The correlation coefficient $(r)$ between TKV slope and eGFR slope is significant $(p=0.0385)$ 
Fig. 3 Individual TKV data and the age at measurement are plotted and connected according to chronic kidney disease (CKD) stages. Finally measured eGFR was used to indicate the CKD stage category

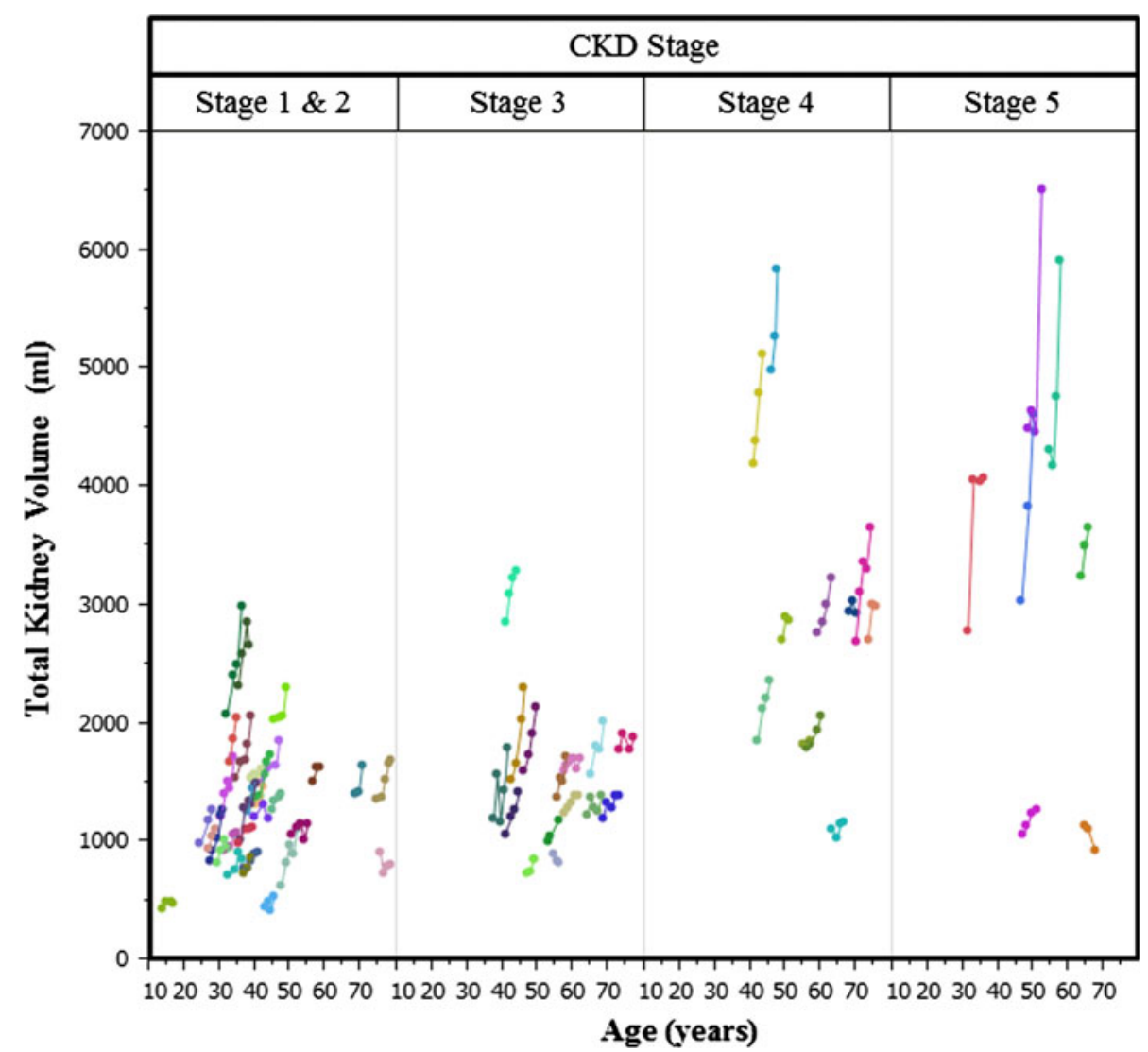

CRISP studies [4, 5, 14-16]. Among adjusted TKV parameters, log-TKV correlated with eGFR most significantly. As the CRISP study showed that TKV increased exponentially and GFR decreased linearly [4], it is reasonable that $\log$-TKV correlates with kidney function better than the other adjusted TKV parameters [14].

Final eGFR but not baseline eGFR correlated with the eGFR slope. This observation is in agreement with our previous report [10], in which the eGFR slope had no correlation with baseline eGFR. The kidney function remains well preserved for many years but decreases rapidly at a later stage $[1,17]$. This characteristic profile of renal function progression is explained by a compensatory adjustment for the loss of GFR. Compensatory adjustments make the decline in GFR slow or close to zero until certain stages [1]. GFR is maintained within the normal range despite decreased renal plasma flow in children and young adult patients with ADPKD [18-20]. In early stages, the decrease in renal plasma flow due to structural distortion in ADPKD is partially compensated for by an increased glomerular filtration fraction to renal plasma flow, but these adaptations eventually prove inadequate and kidney function starts to decline at a faster rate [21]. Those observations and hyperfiltration hypothesis are collectively in accordance with the present finding that the eGFR slope becomes more negative as eGFR decreases (Table 2).
The eGFR slope is relatively constant in relation to age (Fig. 4b). In our previous study, changes of reciprocal creatinine in 106 patients plotted against age showed that the progression patterns of renal function deterioration were different among patients [10]. Individual variation in renal functional progression might be a parallel characteristic to the wide distribution of kidney size growth, as shown in Fig. 3. Due to individual differences, the mean yearly change in eGFR (eGFR slope) as a whole patient group seemed to be constant, at least after $\sim 30$ years of age.

TKV increases each year in most patients with ADPKD (Fig. 3), but no significant correlation between age and TKV was seen in the present study (Table 3). Similar findings were reported in the CRISP study [4]. The reason for this insignificant correlation between TKV and age is probably the wide individual variation in TKV. It is interesting to note that the TKV slope was constant at all ages, but the \% TKV slope and log-TKV slope decreased as age advanced (Table 3; Fig. 5d). This finding has already been reported with the slopes expressed as a percent per year being significantly lower in the older age group $(p=0.02)$ [4]. The mechanism of this saturation-like phenomenon is speculated as follows - the rate of kidney volume enlargement ( $\mathrm{ml} /$ year) is constant throughout life (Table 3), but the growth rate (\%/year) becomes lower 
Table 2 Functional and volume parameters in relation to chronic kidney disease (CKD) stages according to the final measurement of the estimated glomerular filtration rate (eGFR)

\begin{tabular}{|c|c|c|c|c|c|}
\hline & \multicolumn{4}{|c|}{ CKD stage according to the final eGFR $\left(\mathrm{ml} / \mathrm{min} / 1.73 \mathrm{~m}^{2}\right)$ measurement } & \multirow[t]{2}{*}{$p$ value } \\
\hline & $\begin{array}{l}\text { Stages } 1 \text { and } 2 \\
\geq 60\end{array}$ & $\begin{array}{l}\text { Stage } 3 \\
59-30\end{array}$ & $\begin{array}{l}\text { Stage } 4 \\
29-15\end{array}$ & $\begin{array}{l}\text { Stage } 5 \\
<15\end{array}$ & \\
\hline$N$ (men:woman) & $31(10: 21)$ & $15(5: 10)$ & $11(3: 8)$ & $7(3: 4)$ & \\
\hline Observation period (months) & $40.2(11.5)$ & $42.3(10.2)$ & $34.5(11.9)$ & $40.0(9.1)$ & NS \\
\hline Baseline age (years) & $39.8(13.7)$ & $53.3(11.0)$ & $56.4(11.3)$ & $50.7(11.4)$ & $<0.01$ \\
\hline Systolic BP on treatment $(\mathrm{mmHg})$ & $118.9(10.6)$ & $133.2(11.3)$ & $133.5(19.4)$ & $137.1(17.7)$ & $<0.01$ \\
\hline Diastolic BP on treatment $(\mathrm{mmHg})$ & $77.2(6.6)$ & $81.0(4.9)$ & $80.3(10.2)$ & $82.3(11.3)$ & NS \\
\hline Urine protein excretion $\left(\mathrm{mg} / \mathrm{day} / 1.73 \mathrm{~m}^{2}\right)$ & $62.3(96.1)$ & $124.6(119.1)$ & $223.7(267.6)$ & $1,102.7(1,727.6)$ & $<0.01$ \\
\hline \multicolumn{6}{|l|}{ Kidney function } \\
\hline Baseline eGFR $\left(\mathrm{ml} / \mathrm{min} / 1.73 \mathrm{~m}^{2}\right)$ & $82.1(18.2)$ & $52.7(10.7)$ & $33.0(6.7)$ & $21.9(13.5)$ & $<0.01$ \\
\hline Final eGFR $\left(\mathrm{ml} / \mathrm{min} / 1.73 \mathrm{~m}^{2}\right)$ & $82.5(19.4)$ & $46.5(8.6)$ & $24.2(3.1)$ & $7.8(3.7)$ & $<0.01$ \\
\hline eGFR slope $\left(\mathrm{ml} / \mathrm{min} / 1.73 \mathrm{~m}^{2} /\right.$ year$)$ & $0.18(3.47)$ & $-0.74(3.95)$ & $-2.95(2.38)$ & $-3.88(2.89)$ & $<0.01$ \\
\hline Baseline $\operatorname{Ccr}\left(\mathrm{ml} / \mathrm{min} / 1.73 \mathrm{~m}^{2}\right)$ & $114.3(30.7)$ & $85.1(17.8)$ & $48.6(7.0)$ & $39.5(19.4)$ & $<0.01$ \\
\hline Ccr slope $\left(\mathrm{ml} / \mathrm{min} / 1.73 \mathrm{~m}^{2} /\right.$ year $)$ & $-2.11(11.74)$ & $-4.04(3.49)$ & $-4.62(7.96)$ & $-9.59(3.67)$ & NS \\
\hline Baseline 1/Creatinine $(\mathrm{ml} / \mathrm{mg})$ & $143(27)$ & $103(20)$ & $70(15)$ & $42(19)$ & $<0.01$ \\
\hline \multicolumn{6}{|l|}{ Kidney volume } \\
\hline Baseline TKV (ml) & $1,192.0(457.9)$ & $1,394.3(499.9)$ & $2,693.0(1,112.8)$ & $2,871.4(1,362.4)$ & $<0.01$ \\
\hline Final TKV (ml) & $1,440.9(576.7)$ & $1,689.1(618.4)$ & $3,103.7(1,377.2)$ & $3,855.3(2,129.5)$ & $<0.01$ \\
\hline TKV slope (ml/year) & $73.8(51.8)$ & $75.0(68.0)$ & $148.6(146.9)$ & $279.6(234)$ & $<0.01$ \\
\hline$\%$ TKV slope (\%/year) & $6.25(3.86)$ & $5.16(4.74)$ & $4.80(3.14)$ & $7.69(7.09)$ & NS \\
\hline log-TKV slope (ml/year) & $0.0240(0.0140)$ & $0.0244(0.0260)$ & $0.0116(0.0268)$ & $0.0273(0.0277)$ & NS \\
\hline Baseline ht-TKV (ml/m) & $724.7(279.3)$ & $862.1(268.6)$ & $1,681.6(718.7)$ & $1,661.8(787.9)$ & $<0.01$ \\
\hline Baseline bs-TKV $\left(\mathrm{ml} / \mathrm{m}^{2}\right)$ & $714.2(267.4)$ & $890.4(257.0)$ & $1,729.0(764.8)$ & $1,623.5(784.9)$ & $<0.01$ \\
\hline Baseline log-TKV $(\log [\mathrm{ml}])$ & $3.044(0.1759)$ & $3.109(0.1600)$ & $3.396(0.1825)$ & $3.402(0.257)$ & $<0.01$ \\
\hline
\end{tabular}

Numbers are the mean and standard deviation (in parentheses).

Slopes are calculated by regression analysis of each patient. Urine protein excretion and Ccr were measured from 24-h urine. CKD stage 1 and 2 are combined. $p$ values were calculated by ANOVA

$B P$ blood pressure, $C K D$ chronic kidney disease, eGFR glomerular filtration rate estimated by Japanese MDRD equation, $C c r$ creatinine clearance, $T K V$ total kidney volume, $h t-T K V$ TKV divided by height $(\mathrm{m}), b s-T K V$ TKV divided by body surface area $\left(\mathrm{m}^{2}\right), \log -T K V \log -$ converted TKV

Table 3 Correlation coefficient $(r)$ between age and kidney volume, function and their slopes

\begin{tabular}{|c|c|c|c|c|c|}
\hline \multicolumn{3}{|c|}{$r$ between parameters and age at final measurement } & \multicolumn{3}{|c|}{$r$ between each parameter slope and age at final measurement } \\
\hline & $r$ & $p$ value & & $r$ & $p$ value \\
\hline TKV (ml) & 0.1264 & NS & TKV slope (ml/year) & -0.0979 & NS \\
\hline$\%$ TKV (\%/year) & - & - & $\%$ TKV slope (\%/year) & -0.3923 & $<0.01$ \\
\hline ht-TKV (ml/m) & 0.1526 & NS & ht-TKV slope (ml/m/year) & -0.0945 & NS \\
\hline bs-TKV $\left(\mathrm{ml} / \mathrm{m}^{2}\right)$ & 0.1894 & NS & bs-TKV slope $\left(\mathrm{ml} / \mathrm{m}^{2} /\right.$ year $)$ & -0.0545 & NS \\
\hline $\log -\mathrm{TKV}(\log [\mathrm{ml}])$ & 0.1774 & NS & $\log$-TKV slope $(\log [\mathrm{ml}] /$ year $)$ & -0.4002 & $<0.01$ \\
\hline $1 / \mathrm{Cre}(\mathrm{ml} / \mathrm{mg})$ & -0.5097 & $<0.001$ & 1/Cre slope $(\mathrm{ml} / \mathrm{mg} /$ year $)$ & -0.1585 & NS \\
\hline eGFR $\left(\mathrm{ml} / \mathrm{min} / 1.73 \mathrm{~m}^{2}\right)$ & -0.6027 & $<0.001$ & eGFR slope $\left(\mathrm{ml} / \mathrm{min} / 1.73 \mathrm{~m}^{2} /\right.$ year$)$ & -0.0809 & NS \\
\hline $\operatorname{Ccr}\left(\mathrm{ml} / \mathrm{min} / 1.73 \mathrm{~m}^{2}\right)$ & -0.436 & $<0.001$ & Ccr slope $\left(\mathrm{ml} / \mathrm{min} / 1.73 \mathrm{~m}^{2} /\right.$ year $)$ & -0.1592 & NS \\
\hline
\end{tabular}

Correlation coefficients $(r)$ are calculated between each parameter and final age.

$T K V$ total kidney volume, $h t-T K V$ TKV divided by height $(\mathrm{m}), b s-T K V$ TKV divided by body surface area $\left(\mathrm{m}^{2}\right), \log$ - $T K V \log$-converted TKV, $C r$ creatinine, $e G F R$ estimated glomerular filtration rate by Japanese MDRD equation, $C c r$ creatinine clearance 
(A) Estimated GFR

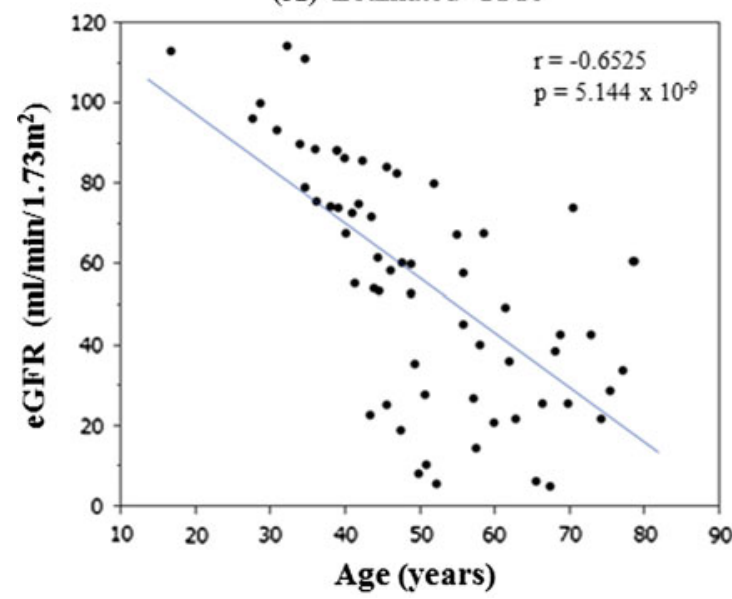

(B) Estimated GFR Slope

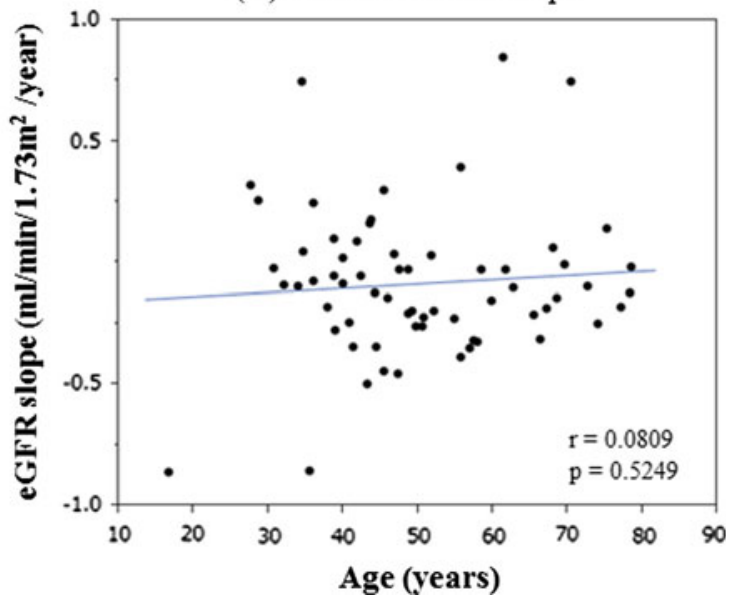

Fig. 4 a Correlation coefficient $(r)$ between eGFR and age is highly significant. Age and eGFR are those measured at the final time. b There was no significant correlation coefficient $(r)$ between age and the slope of eGFR. Age is at the final measurement

(A) Age and TKV

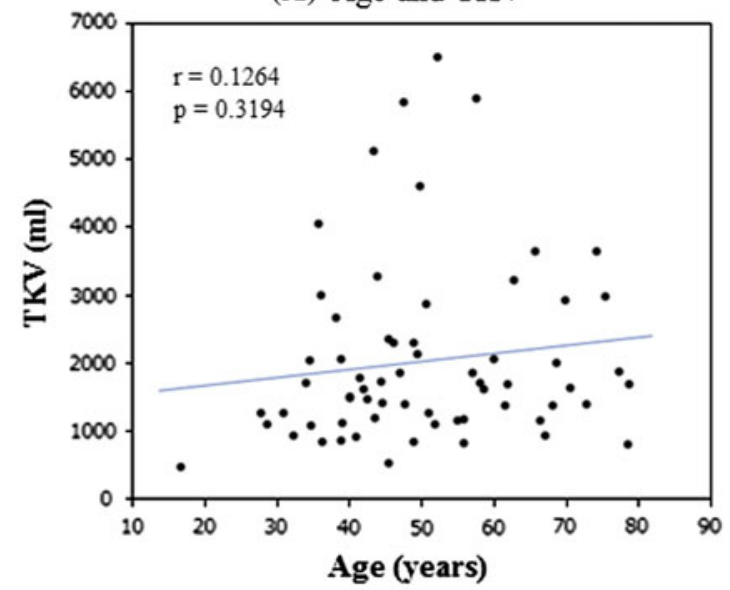

(C) Age and TKV slope

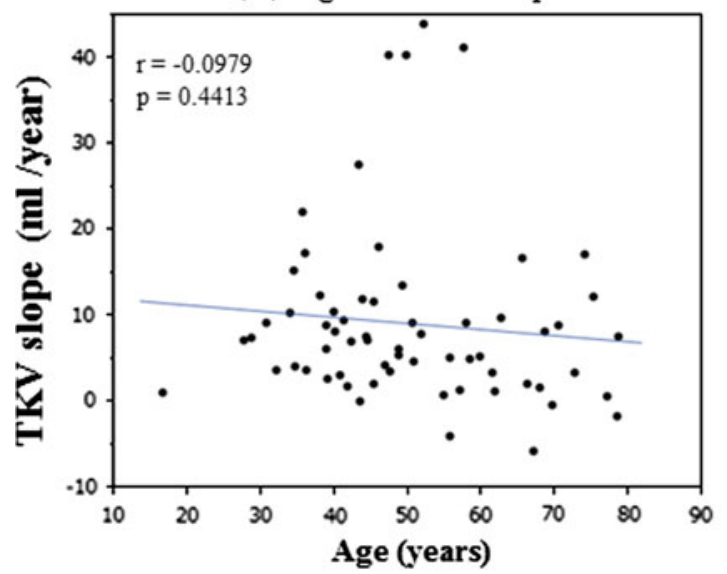

Fig. 5 The correlation coefficients $(r)$ between age and TKV $\mathbf{a}$ and between age and log-TKV $\mathbf{b}$ are not significant. $\mathbf{c}$ The TKV slope tends to decrease as age advances, but $r$ between age and TKV slope
(B) Age and log-TKV

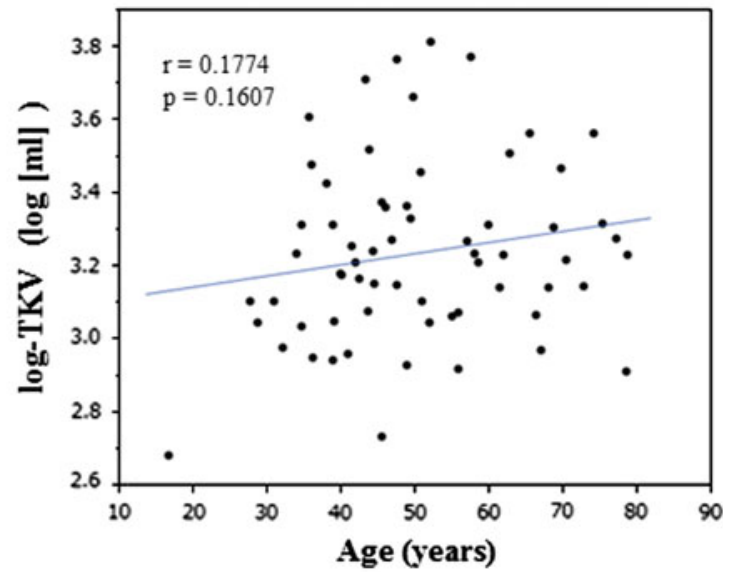

(D) Age and log-TKV slope

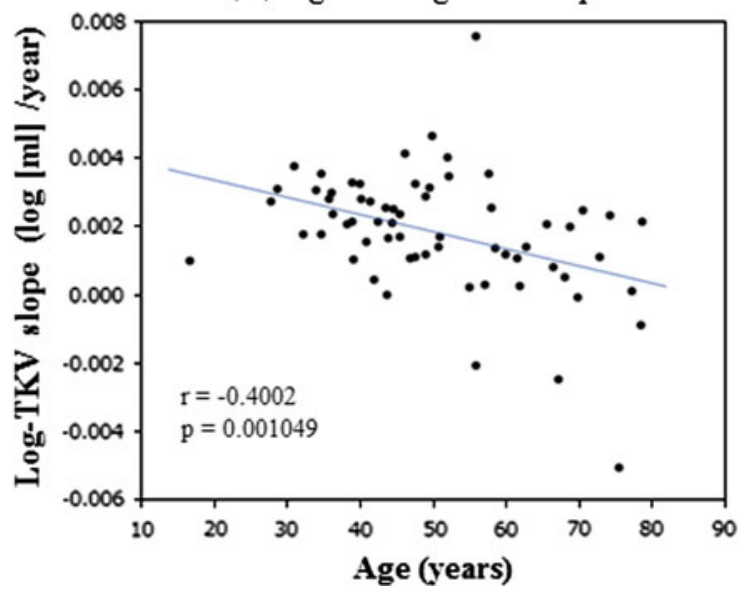

is not significant. d The log-TKV slope decreased significantly as age increased. The $r$ between age and log-TKV slope is significant $(p<0.01)$. Age, TKV and log-TKV are final measurements 
because the denominator (kidney volume) increases every year. The same explanation is applicable to log-converted kidney volume.

The highly significant correlation between baseline as well as final TKV and TKV slope is an obvious result of a large kidney being the consequence of a rapid increase in kidney volume. Although genotype was not determined in the present study, it is known that faster growth is generally associated with PKD1 genotype [4]. A large kidney volume was associated with a more rapid declining slope of iothalamate-measured GFR as well as of eGFR in the present study (Fig. 2a), indicating that a large kidney volume is associated with decreased kidney function [4]. Recently, Chapman et al. reported that baseline ht-TKV $\geq 600 \mathrm{cc} / \mathrm{m}$ predicted the risk of developing renal insufficiency within 8 years [5]. The present study is not long enough to quantitatively predict the risk of renal insufficiency but supports the view that TKV is a prognostic biomarker in ADPKD.

In summary, this study confirmed that TKV is a clinically meaningful surrogate marker in ADPKD because it correlates with kidney function and predicts functional disease progression. Patients with larger TKV are at higher risk of developing ESRD.

\section{Limitations of this study}

Kidney function was not measured directly, such as by inulin clearance. Twenty-four-hour urine creatinine clearance is known to have a relatively large variance due to method imprecision and tubular creatinine secretion [22]. eGFR and reciprocal creatinine are affected by non-GFR factors such as creatinine production and tubular secretion. The patient number is limited and the observation period is not long enough to predict disease progression.

Acknowledgments This study was supported in part by a Grant-inAid for Progressive Renal Diseases Research from the Ministry of Health, Labor and Welfare of Japan.

Conflict of interest All the authors have declared no competing interests.

Open Access This article is distributed under the terms of the Creative Commons Attribution License which permits any use, distribution, and reproduction in any medium, provided the original author(s) and the source are credited.

\section{References}

1. Grantham JJ, Chapman AB, Torres VE. Volume progression in autosomal dominant polycystic kidney disease: the major factor determining clinical outcomes. Clin J Am Soc Nephrol. 2006; 1:148-57.

2. Torres VE, Harris PC, Pirson Y. Autosomal dominant polycystic kidney disease. Lancet. 2007;369:1287-301.
3. Higashihara E, Nutahara K, Kojima M, Tamakoshi A, Ohno Y, Sasaki H, Kurokawa K. Prevalence and renal prognosis of diagnosed autosomal dominant polycystic kidney disease in Japan. Nephron. 1998;80:421-7.

4. Grantham JJ, Torres VE, Chapman AB, Guay-Woodford LM, Bae KT, King BF Jr, Wetzel LH, Baumgarten DA, Kenney PJ, Harris PC, Klahr S, Bennett WM, Hirschman GN, Meyers CM, Zhang X, Zhu F, Miller JP, CRISP Investigators. Volume progression in polycystic kidney disease. N Engl J Med. 2006;354: 2122-30.

5. Chapman AB, Bost JE, Torres VE, Guay-Woodford L, Bae KT, Landsittel D, Li J, King BF, Martin D, Wetzel LH, Lockhart ME, Harris PC, Moxey-Mims M, Flessner M, Bennett WM, Grantham $\mathrm{JJ}$. Kidney volume and functional outcomes in autosomal dominant polycystic kidney disease. Clin J Am Soc Nephrol. 2012;7:479-86.

6. Perico N, Antiga L, Caroli A, Ruggenenti P, Fasolini G, Cafaro M, Ondei P, Rubis N, Diadei O, Gherardi G, Prandini S, Panozo A, Bravo RF, Carminati S, De Leon FR, Gaspari F, Cortinovis M, Motterlini N, Ene-Iordache B, Remuzzi A, Remuzzi G. Sirolimus therapy to halt progression of ADPKD. J Am Soc Nephrol. 2010; 21:1031-40.

7. Walz G, Budde K, Mannaa M, Nürnberger J, Wanner C, Sommerer C, Kunzendorf U, Banas B, Hörl WH, Obermüller N, Arns W, Pavenstädt H, Gaedeke J, Büchert M, May C, Gschaidmeier $\mathrm{H}$, Kramer S, Eckardt KU. Everolimus in patients with autosomal dominant polycystic kidney disease. N Engl J Med. 2010; 363:830-40.

8. Serra AL, Poster D, Kistler AD, Karauer F, Raina S, Young J, Rentsch KM, Spanaus KS, Senn O, Kristanto P, Scheffel H, Weishaupt D, Wüthrich RP. Sirolimus and kidney growth in autosomal dominant polycystic kidney disease. N Engl J Med. 2010;363:820-9.

9. Kistler AD, Poster D, Krauer F, Weishaupt D, Raina S, Senn O, Binet I, Spanaus K, Wüthrich RP, Serra AL. Increases in kidney volume in autosomal dominant polycystic kidney disease can be detected within 6 months. Kidney Int. 2009;75:235-41.

10. Higashihara E, Horie $S$, Muto $S$, Mochizuki T, Nishio $S$, Nutahara K. Renal disease progression in autosomal dominant polycystic kidney disease. Clin Exp Nephrol. 2012;16:622-8.

11. Matsuo S, Imai E, Horio M, Yasuda $Y$, Tomita $K$, Nitta $K$, Yamagata K, Tomino Y, Yokoyama H, Hishida A, Collaborators developing the Japanese equation for estimated GFR. Revised equations for estimated GFR from serum creatinine in Japan. Am J Kidney Dis. 2009;53:982-92.

12. Work Group and evidence review team membership. K/DOQI clinical practice guidelines on chronic kidney disease. Am J Kidney Dis. 2002;39:S1-216.

13. Bae KT, Commean PK, Lee J. Volumetric measurement of renal cysts and parenchyma using MRI: phantoms and patients with polycystic kidney disease. J Comput Assist Tomogr. 2000;24: 614-9.

14. Torres VE, Grantham JJ, Chapman AB, Mrug M, Bae KT, King BF Jr, Wetzel LH, Martin D, Lockhart ME, Bennett WM, Moxey-Mims M, Abebe KZ, Lin Y, Bost JE, Consortium for Radiologic Imaging Studies of Polycystic Kidney Disease (CRISP). Potentially modifiable factors affecting the progression of autosomal dominant polycystic kidney disease. Clin J Am Soc Nephrol. 2011;6:640-7.

15. Torres VE, King BF, Chapman AB, Brummer ME, Bae KT, Glockner JF, Arya K, Risk D, Felmlee JP, Grantham JJ, GuayWoodford LM, Bennett WM, Klahr S, Meyers CM, Zhang X, Thompson PA, Miller JP, Consortium for Radiologic Imaging Studies of Polycystic Kidney Disease (CRISP). Magnetic resonance measurements of renal blood flow and disease progression in autosomal dominant polycystic kidney disease. Clin J Am Soc Nephrol. 2007;2:112-20. 
16. Chapman AB, Guay-Woodford LM, Grantham JJ, Torres VE, Bae KT, Baumgarten DA, Kenney PJ, King BF Jr, Glockner JF, Wetzel LH, Brummer ME, O’Neill WC, Robbin ML, Bennett WM, Klahr S, Hirschman GH, Kimmel PL, Thompson PA, Miller JP, Consortium for Radiologic Imaging Studies of Polycystic Kidney Disease cohort. Renal structure in early autosomaldominant polycystic kidney disease (ADPKD): The consortium for radiologic imaging studies of polycystic kidney disease (CRISP) cohort. Kidney Int. 2003;64:1035-45.

17. Franz KA, Reubi FC. Rate of functional deterioration in polycystic kidney disease. Kidney Int. 2009;23:526-9.

18. Wong H, Vivian L, Weiler G, Filler G. Patients with autosomal dominant polycystic kidney disease hyperfiltrate early in their disease. Am J Kidney Dis. 2004;43:624-8.

19. Meijer E, Rook M, Tent H, Navis G, van der Jagt EJ, de Jong PE, Gansevoort RT. Early renal abnormalities in autosomal dominant polycystic kidney disease. Clin J Am Soc Nephrol. 2010;5: 1091-8.
20. Helal I, Reed B, McFann K, Yan XD, Fick-Brosnahan GM, Cadnapaphornchai M, Schrier RW. Glomerular hyperfiltration and renal progression in children with autosomal dominant polycystic kidney disease. Clin J Am Soc Nephrol. 2011;6: 2439-43.

21. Brenner BM. Nephron adaptation to renal injury or ablation. Am J Physiol. 1985;249:F324-37.

22. Rule AD, Torres VE, Chapman AB, Grantham JJ, Guay-Woodford LM, Bae KT, Klahr S, Bennett WM, Meyers CM, Thompson PA, Miller JP, CRISP Consortium. Comparison of methods for determining renal function decline in early autosomal dominant polycystic kidney disease: the consortium of radiologic imaging studies of polycystic kidney disease cohort. J Am Soc Nephrol. 2006; 17:854-62. 